Applied Physics A manuscript No.

(will be inserted by the editor)

\title{
Electronic structure and dynamics of optically excited single-wall carbon nanotubes
}

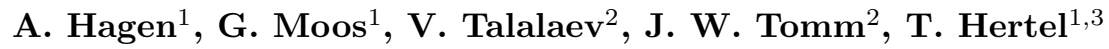 \\ 1 Department of Physical Chemistry, Fritz-Haber-Institut der Max-Planck-Society, Faradayweg 4-6, D-14195 Berlin, Germany \\ ' 2 Max-Born-Institut für Nichtlineare Optik und Kurzzeitspektroskopie, Max Born Str. 2 A, D-12489 Berlin, Germany \\ 3 Department of Physics and Astronomy, Vanderbilt University, Nashville, Tennessee, USA
}

Received: date / Revised version: date

\begin{abstract}
We have studied the electronic structure and charge-carrier dynamics of individual single-wall carbon nanotubes (SWNTs) and nanotube ropes using optical and electron-spectroscopic techniques. The electronic structure of semiconducting SWNTs in the band-gap region is analyzed using near-infrared absorption spectroscopy. A semi-empirical expression for $E_{11}^{S}$ transition energies, based on tight-binding calculations is found to give striking agreement with experimental data. Time-resolved PL from dispersed SWNT-micelles shows a decay with a time constant of about 15 ps. Using time-resolved photoemission we also find that the electron-phonon $(e-p h)$ coupling in metallic tubes is characterized by a very small $e$-ph mass-enhancement of 0.0004. Ultrafast electronelectron scattering of photo-excited carriers in nanotube ropes is finally found to lead to internal thermalization ' of the electronic system within about $200 \mathrm{fs}$.
\end{abstract}

\section{Introduction}

The electronic properties of single-wall carbon nanotubes (SWNTs) and specifically of semiconducting SWNTs hold promise for application in a variety of optical devices such as light-emitting- 1] and photo-sensitive diodes [2], for nonlinear optical- or light harvesting materials and more. The electronic structure and the dynamics of optically excited states in SWNTs naturally plays a decisive role for the operation of such devices. In particular the scattering times of photoexcited carriers with electrons, phonons and defects as well as the competition of radiative with non-radiative decay processes are of great interest for a better understanding of electronic transport phenomena in optical devices $3,4,5,6,7,8,9,10,11$, 12, 13, 14.

Here, we make use of the recent development of techniques for the exfoliation of SWNTs from nanotuberopes which provides the basis for a detailed investigation of optical properties and excitations in individ- ual SWNTs using near-infrared (NIR) absorption spectroscopy or spectrofluorimetric techniques [15,16. These advances also allow to address questions of fundamental interest such as the character of optical excitations in systems with reduced dimensionality. Binding energies of Wannier-type excitons, for example, are predicted to depend strongly on the dimensionality and the lowest state is expected to diverge towards infinite binding energy as the system becomes truly one-dimensional [17. 18. It has previously been speculated that some optical transitions in individual SWNTs are excitonic, but so far, the reported evidence is not conclusive. Here, we will discuss the character of optical excitations in individual SWNTs in further detail. The corresponding transition energies are analyzed using tight-binding (TB) calculations which show that the observed variation of transition energies with tube diameter and chirality can be identified with variations in the TB band-gaps. The decay of optically excited states is determined by the competition of non-radiative and radiative decay processes. These are investigated experimentally using picosecond time-resolved photoluminescence (PL) spectroscopy.

We have furthermore studied carrier dynamics in SWNT ropes using femtosecond time-resolved photoemission. A detailed analysis of these experiments allows to determine characteristic timescales of fundamental scattering processes such as electron-electron $(e-e)$ or electronphonon $(e-p h)$ scattering. We find that $e-e$ interactions in bundles of SWNTs give rise to $e$ - $e$ scattering times which strongly increase as the carrier energy approaches the Fermi level. The internal thermalization of the photoexcited electron gas is facilitated by such $e$-e scattering processes and is characterized by a time constant of $200 \mathrm{fs}$. The electron-phonon $(e-p h)$ coupling in metallic tubes finally is found to be extraordinarily weak with a mass enhancement of only 0.0004 . The latter suggests that room temperature $e$-ph scattering times are on the order of $15 \mathrm{ps}$. These results are discussed with respect to the influence of radiative and non-radiative decay pro- 
cesses on the carrier dynamics in individual tubes and tube ropes.

\section{Experimental}

Ultraviolet to visible to near-infrared (UV-VIS-NIR) absorption spectra and time-resolved PL studies were obtained from samples synthesized by the high pressure $\mathrm{CO}$ decomposition technique 19. So called HiPCO material has been purchased from CNI Houston. For absorption experiments, individual SWNTs were isolated from HiPCO material in sodiumdodecylsulfate (SDS) micelles by the technique described in ref. 15. At wavelengths below $1350 \mathrm{~nm}$ the well resolved spectrum from micelles in $\mathrm{H}_{2} \mathrm{O}$ was extended by results from measurements in $\mathrm{D}_{2} \mathrm{O}$. For PL measurements the samples were dispersed using sodium cholate. Time-resolved PL from SWNT cholate micelles is performed using $300 \mathrm{~mW}$ of output from a Tsunami Ti:sapphire with sub-100 fs excitation pulses at a repetition rate of $82 \mathrm{MHz}$ and a wavelength of $795 \mathrm{~nm}$. Detection is done by a Hamamatsu synchroscan streak camera with an IR-enhanced cathode. The time resolution is here found to be slightly better than $10 \mathrm{ps}$. The laser spot size in the $2 \mathrm{~mm}$ long quartz cuvette was $\approx 0.5 \mathrm{~mm}$ in diameter. This allowed to keep pulse fluences at a very low level of $10^{13}$ photons per $\mathrm{cm}^{2}$ or less.

Time-resolved photoemission experiments were here performed with samples consisting of purified nanotube ropes that were produced by the laser vaporization technique (tubes@rice). A Ti:sapphire laser pumped optical parametric amplifier (Coherent) generates tunable femtosecond pulses with wavelengths between $470 \mathrm{~nm}$ and $730 \mathrm{~nm}$. Fundamental and second harmonic beams generated from the OPA output are used as visible pump and UV probe respectively. The fluence for the visible pump and UV probe beams is typically $50 \mu \mathrm{J} \mathrm{cm}^{-2}$ and $5 \mu \mathrm{J} \mathrm{cm}^{-2}$, respectively, which corresponds to a pump power of $10^{9} \mathrm{~W} \mathrm{~cm}^{-2}$. For more details see reference 7.].

\section{Results and discussion}

In Fig. 1]we compare the absorption spectrum from colloidal graphite with spectra from SWNT-ropes as well as from individual exfoliated SWNT-micelles in aqueous solution. The optical density of colloidal graphite increases continuously from the near infrared to the UV range due to the logarithmic singularity in the joint density of states near $5 \mathrm{eV}$ for excitation of free carriers from $\pi$ to $\pi^{*}$ bands 20,21. The optical density in SWNT ropes follows a similar general trend as seen for graphite illustrating the intimate relationship of the electronic structure of these two materials. However, the middle spectrum in Fig. 1 from SWNT ropes also exhibits some pronounced fine structure on top of the continuously

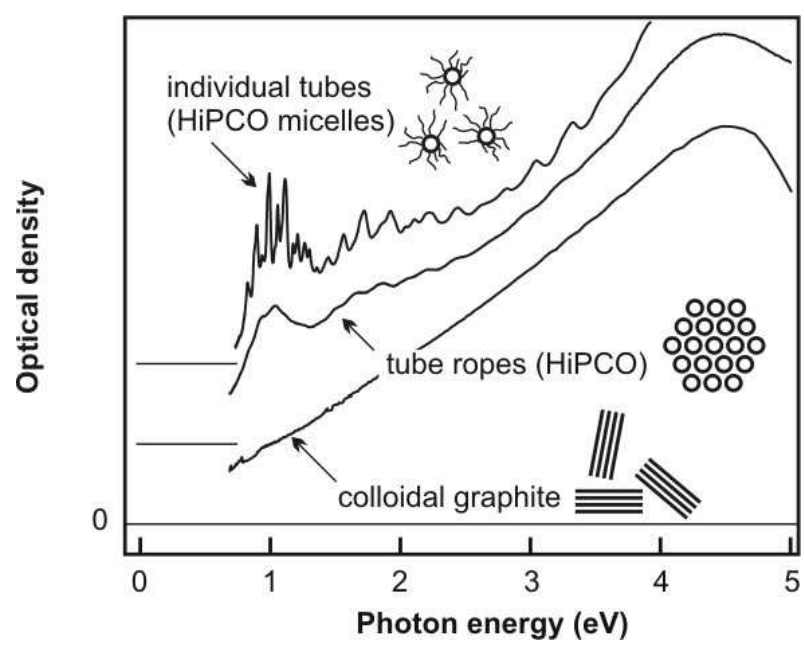

Fig. 1 Comparison of the optical density of colloidal graphite (lower trace), crystalline single-wall carbon nanotube ropes (middle trace) and exfoliated SWNTs in solution.

rising background. These features can be attributed to optical transitions between different sub-bands of semiconducting and metallic SWNTs in the suspension. For tubes with a diameter of approximately $1 \mathrm{~nm}$, absorption features in the range from $0.8 \mathrm{eV}$ to $1.3 \mathrm{eV}$ and features in-between about $1.4 \mathrm{eV}$ and $2.5 \mathrm{eV}$ are associated with $E_{11}^{S}$ and $E_{22}^{S}$ transitions between the first and second $\pi$ sub-bands in semiconduction tubes, respectively. Broad and not clearly resolved features between about $2.0 \mathrm{eV}$ to $3.5 \mathrm{eV}$ are due to $E_{11}^{M}$ transitions between the first sub-bands in metallic tubes. The nomenclature used here for optical transitions is $E_{k l}^{X}$, where $k$ and $l$ refer to the valence and conduction sub-band index, respectively and $X=S, M$ refers to transitions in semiconducting or metallic tubes, respectively. Transitions with $k=l$ are dipole-allowed for light polarized parallel to the tube axis 22 .

If individual SWNTs are combined into ropes one finds that $E_{11}^{S}$ transitions become substantially broadened. The corresponding $100 \mathrm{meV}$ increase of the FWHM of absorption features is indicative for the large contribution of tube-tube interactions to the width of transitions in spectra from SWNT ropes [13. Higher lying $E_{22}^{S}$ and $E_{11}^{M}$ transitions are broad both in the exfoliated and crystalline phase which suggests that intratube relaxation from higher energy states provides an efficient decay channel for tubes in all environments. In the following we will begin the discussion with a detailed analysis of $E_{11}^{S}$ transitions within the first absorption cluster.

\subsection{Analysis of absorption spectra from SWNT-micelles}

In Fig. 2] we have plotted a background corrected absorption spectrum with well-resolved spectral features in the range of the first absorption cluster. To analyze the optical transitions in this energy range in further detail we 

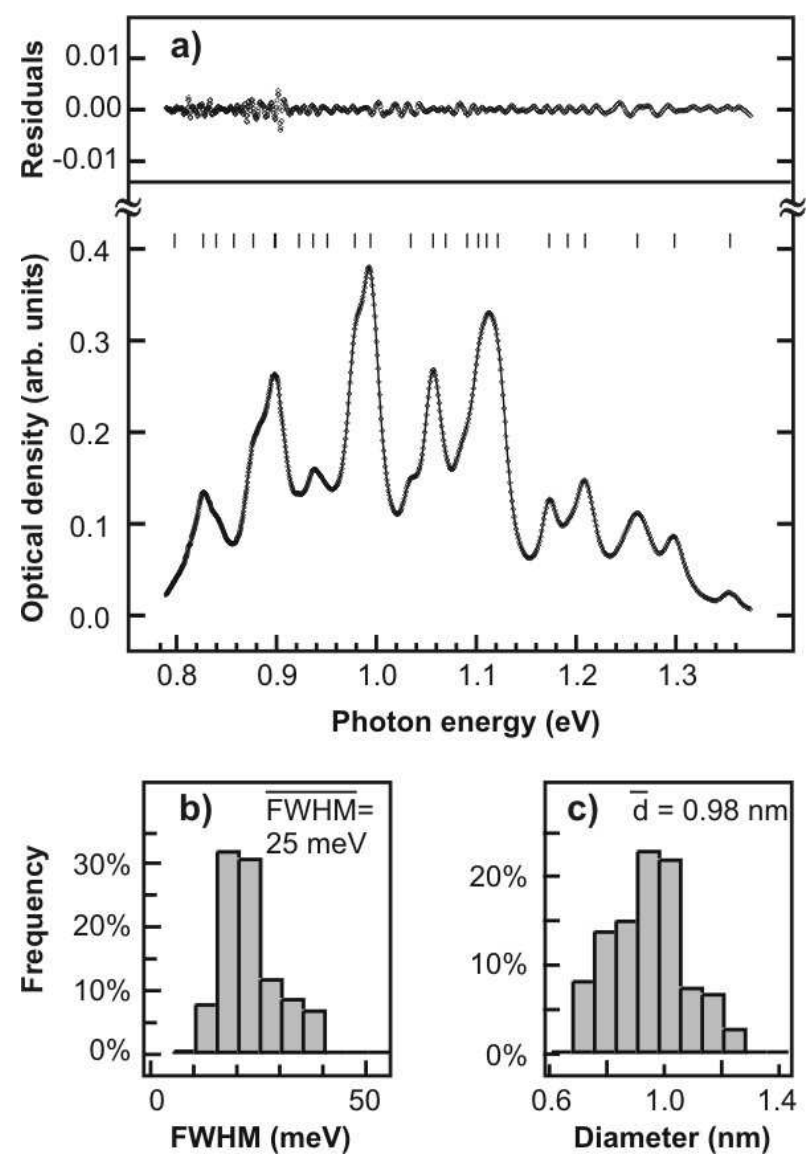

Fig. 2 a) Near-infrared region of the background corrected SWNT micelle absorption spectrum (circles) and multi-peak fit using a linear combination of 24 Voigt profiles (thin line). Individual peak positions are marked by vertical lines above the spectrum. b) the resulting diameter- and c) peak-width distributions for semiconducting SWNTs.

performed a multi-peak fit with a maximum of 24 independent Voigt profiles. The resulting fit is shown in Fig. 2a) as thin line superimposed on the experimental data (dots). The excellent agreement between fit and experiment can be appreciated from the nearly vanishing residuals in the upper part of Fig. 2 2 ). Due to the excellent signal to noise ratio, errors of peak positions are on average only about $1 \mathrm{meV}$. The experimental peak energies obtained here can be found in Table 1 together with transitions obtained from PL measurements [16]. The distribution of peak widths with its mean at $25 \mathrm{meV}$ is shown in Fig. 2b). The contribution of Gaussian and Lorentzian components to the Voigt profiles is about equal. Similar average widths of $23 \mathrm{meV}$ and $25 \mathrm{meV}$ were recently also observed for band-gap PL from single SWNTs dispersed on a surface or SWNT micelle ensembles in solution, respectively [16,23].

A comparison of peak positions obtained here with those reported by Bachilo et al. for PL measurements from SWNT-micelles dispersed in $\mathrm{D}_{2} \mathrm{O}$ [16] yields ex-

\footnotetext{
${ }^{1}$ Values taken from ref. [15] are redshifted by $3 \mathrm{meV}$.
}

Table 1 Comparison of the position of spectral absorption features obtained experimentally and from the semiempirical, TB band-structure based model.

\begin{tabular}{llll}
\hline Tube type & $d(\mathrm{~nm})$ & $h \nu_{11}^{\text {expt }}(\mathrm{eV})$ & $h \nu_{11}^{\text {calc }}(\mathrm{eV})$ \\
\hline$(5,4)$ & 0.62 & $1.485^{1}$ & 1.480 \\
$(6,4)$ & 0.69 & $1.417^{1}$ & 1.421 \\
$(9,1)$ & 0.76 & $1.354 \pm 0.001$ & 1.357 \\
$(8,3)$ & 0.78 & $1.298 \pm 0.001$ & 1.302 \\
$(6,5)$ & 0.76 & $1.261 \pm 0.001$ & 1.260 \\
$(7,5)$ & 0.83 & $1.208 \pm 0.001$ & 1.208 \\
$(11,0)$ & 0.87 & $1.190 \pm 0.001$ & 1.187 \\
$(10,2)$ & 0.88 & $1.172 \pm 0.001$ & 1.170 \\
$(9,4)$ & 0.91 & $1.120 \pm 0.007$ & 1.123 \\
$(8,4)$ & 0.84 & $1.109 \pm 0.002$ & 1.108 \\
$(7,6)$ & 0.89 & $1.100 \pm 0.001$ & 1.097 \\
$(9,2)$ & 0.81 & $1.089 \pm 0.008$ & 1.095 \\
$(12,1)$ & 0.99 & $1.067 \pm 0.001$ & 1.053 \\
$(8,6)$ & 0.97 & $1.054 \pm 0.001$ & 1.053 \\
$(11,3)$ & 1.01 & $1.032 \pm 0.001$ & 1.032 \\
$(10,3)$ & 0.94 & $0.989^{1}$ & 0.985 \\
$(10,5)$ & 1.05 & $0.989^{1}$ & 0.988 \\
$(9,5)$ & 0.97 & $0.991 \pm 0.001$ & 0.988 \\
$(11,1)$ & 0.91 & $0.979^{1}$ & 0.976 \\
$(8,7)$ & 1.03 & $0.976 \pm 0.001$ & 0.971 \\
$(13,2)$ & 1.12 & $0.948 \pm 0.003$ & 0.947 \\
$(9,7)$ & 1.10 & $0.933 \pm 0.001$ & 0.935 \\
$(12,4)$ & 1.14 & $0.919 \pm 0.001$ & 0.924 \\
$(10,6)$ & 1.11 & $0.895^{1}$ & 0.889 \\
$(11,4)$ & 1.07 & $0.896 \pm 0.001$ & 0.897 \\
$(12,2)$ & 1.04 & $0.895 \pm 0.002$ & 0.896 \\
$(11,6)$ & 1.18 & $0.884^{1}$ & 0.885 \\
$(9,8)$ & 1.17 & $0.873 \pm 0.001$ & 0.873 \\
$(15,1)$ & 1.23 & $0.867^{1}$ & 0.871 \\
$(13,5)$ & 1.28 & $0.835^{1}$ & 0.836 \\
$(10,8)$ & 1.24 & $0.836 \pm 0.007$ & 0.842 \\
$(12,5)$ & 1.20 & $0.826^{1}$ & 0.820 \\
$(13,3)$ & 1.17 & $0.823 \pm 0.001$ & 0.826 \\
$(10,9)$ & 1.31 & $0.794 \pm 0.004$ & 0.792 \\
\hline
\end{tabular}

traordinary agreement if a $3 \mathrm{meV}$ blue-shift of the fluorimetric data is accounted for. The previously observed redshift of luminescence data of a few meV [15] here appears to be overcompensated by differences in transition energies in different environments, i.e. in heavy and light water. The peak assignment adopted here is practically identical to that previously discussed in ref. [13] as 'scenario I' and is guided by the energetic order of peaks in fluorimetric data of Bachilo et al. [16]. In cases where no peaks are reported from PL data, our assignment was guided by the order of peaks expected from the semi-empirical TB band-structure model discussed below. If the spectral weight of individual peaks in the absorption spectrum is combined with the assignment to specific tube types, i.e. tube diameters, we can also calculate the diameter distribution of semiconducting tubes in the present sample which is reproduced in Fig. 2r). The mean diameter of $0.98 \mathrm{~nm}$ obtained here is in good agreement with that recently obtained by a luminescence 
study $0.96 \mathrm{~nm} 16$ and Raman studies, with $1.13 \mathrm{~nm} 24$ and $0.98 \mathrm{~nm}$ 25]. The diameter distribution obtained in ref. 13 is at variance with that obtained here, apparently because the statistical weight of individual tube types varies if the JDOS - as in a previous study [13] - or Voigt profiles are used to fit experimental spectra. The probability distribution of the chiral vector map resulting from the above assignment and fit shows a slight preference for armchair tubes.

Next, we will attempt a quantitative discussion of observed transition energies by comparison with energies predicted by a refined version 26.13 of the original TB band-structure model 27, 28. The TB calculations used here explicitly include the crucial diameter and chirality dependence of the TB hopping integral $\gamma_{0}$ without which any such comparison would be useless. The refined TB-model leads to more pronounced oscillations of band-gaps with tube diameter than predicted by the earlier zone folding schemes because the latter used to neglect mutual realignment of $p_{z}$ orbitals on the curved graphene sheet. However, the magnitude of $\gamma_{0}^{\infty}$, the TB hopping integral for the uncurved graphene sheet, is frequently used as a free parameter and there is no general agreement on the appropriate value that should be used for optical excitations. Scanning tunneling spectroscopy of individual SWNTs yields $\gamma_{0}^{\infty}$ values between $2.45 \mathrm{eV}$ and $2.7 \mathrm{eV}$ 29 30, while an analysis of optical experiments suggest that values between $2.65 \mathrm{eV}$ and $3.0 \mathrm{eV}$ are more appropriate (see, for example, refs. [7] and [31]). Resonant Raman spectra are frequently analyzed using a hopping integral around $3.0 \mathrm{eV}[32$.

If experimental peak positions are here fit by the oneparameter TB model one obtains best agreement with calculated gaps if $\gamma_{0}^{\infty}=3.450 \mathrm{eV}$. The resulting residuals can be characterized by a $25 \mathrm{meV}$ root mean square (RMS) and are plotted as a function of tube diameter in Fig. (3). The steep slope of the straight line fit to residuals clearly illustrates that the one-parameter TB model cannot account for the observed diameter dependence of transition energies. This deficiency can be removed by the addition of a number of empirical corrective terms which give successively better agreement with experiment and thereby also allow to discuss the possible physical origin of remaining discrepancies. Previously, a first order correction was introduced in the form of an empirical offset parameter $\Omega$ [13] which, in combination with a variable $\gamma_{0}^{\infty}$, allow to skew the diameter dependence of calculated transition energies (see Fig. 3b)). Within this two parameter model, best agreement is obtained for $\gamma_{0}^{\infty}=3.059 \mathrm{eV}$ and $\Omega=0.120 \mathrm{eV}$. However, the resulting residuals exhibit a clear correlation with the tube chiral angle $\theta(n, m)=\arcsin \left(\sqrt{3} m /\left(2 \cdot \sqrt{n^{2}+n \cdot m+m^{2}}\right)\right)$ as seen in Fig. 35). The second order correction thus includes a term which varies with the chiral angle as $A \cdot \cos (6 \cdot \theta(n, m))$ (see solid line in Fig. 35). At this point the root mean square deviation of experimental and calculated transition energies is only $6 \mathrm{meV}\left(\gamma_{0}^{\infty}=\right.$
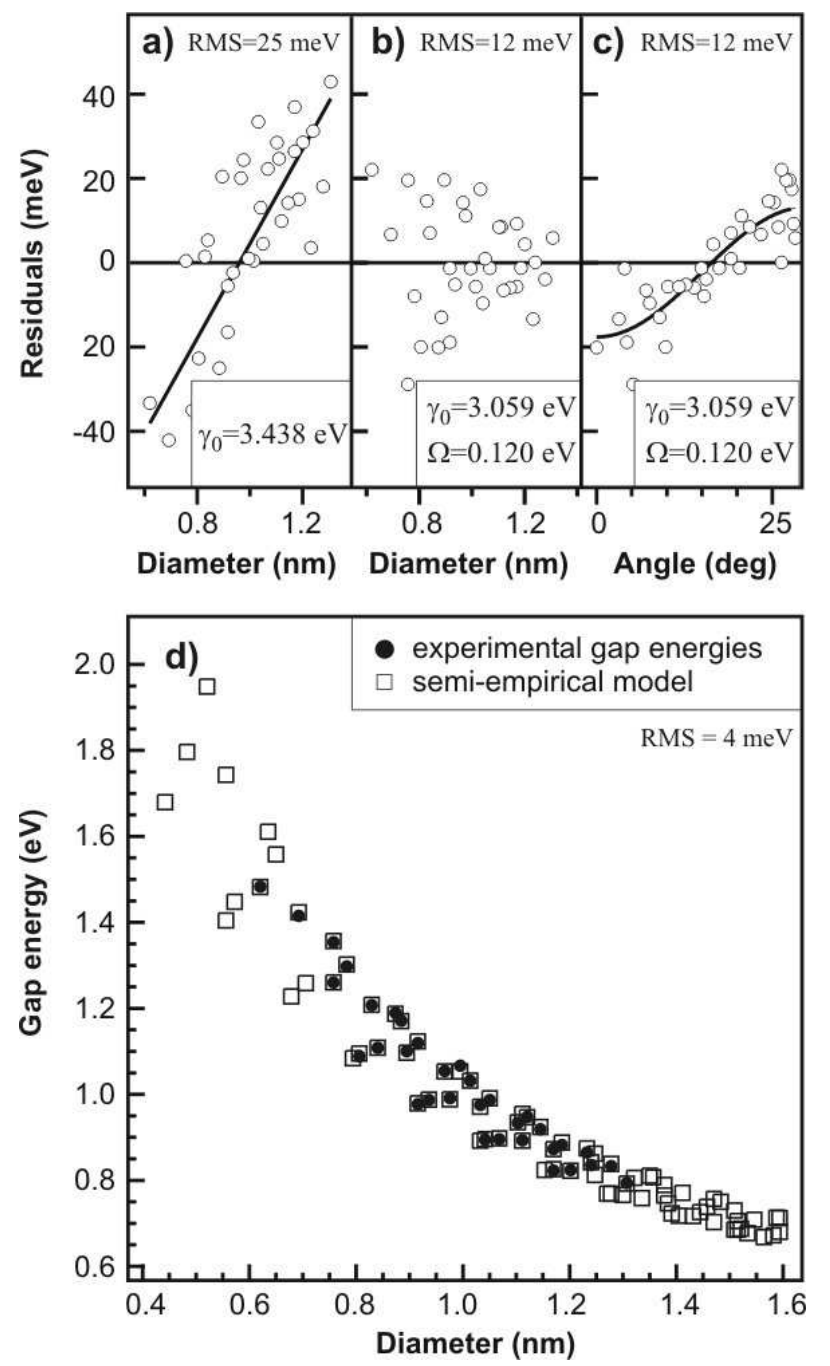

Fig. 3 a-c) Residuals from a fit of experimental data with transition energies from a semi-empirical TB band-structure model. The agreement between experiment and model becomes increasingly better if a phenomenological band offset parameter as well as a chirality correction are included. The straight line in a) is the linear regression and the solid curve in c) represents the contribution from the phenomenological chirality correction. Experimental transition energies are compared in d) with predictions from the four parameter semi-empirical model. The root mean square deviation (RMS) of experimental and model transition energies is also indicated.

$3.061 \mathrm{eV}, \Omega=0.118 \mathrm{eV}, A=-0.0154 \mathrm{eV})$. The last corrective term $B \cdot d(n, m) \cdot \cos (6 \cdot \theta(n, m))$ is included to partially remove a combined correlation of residuals with diameter $d$ and chiral angle $\theta$. The phenomenological four parameter model describing optical transition energies $E_{11}^{S}$ in the band-gap region is finally given by:

$$
\begin{aligned}
E_{11}^{\mathrm{optS}}(n, m) & =\gamma_{0}^{\infty} \cdot \epsilon_{11}^{\mathrm{TB}}(n, m) \\
& +(A+B \cdot d(n, m)) \cos [6 \cdot \theta(n, m)] \\
& +\Omega
\end{aligned}
$$




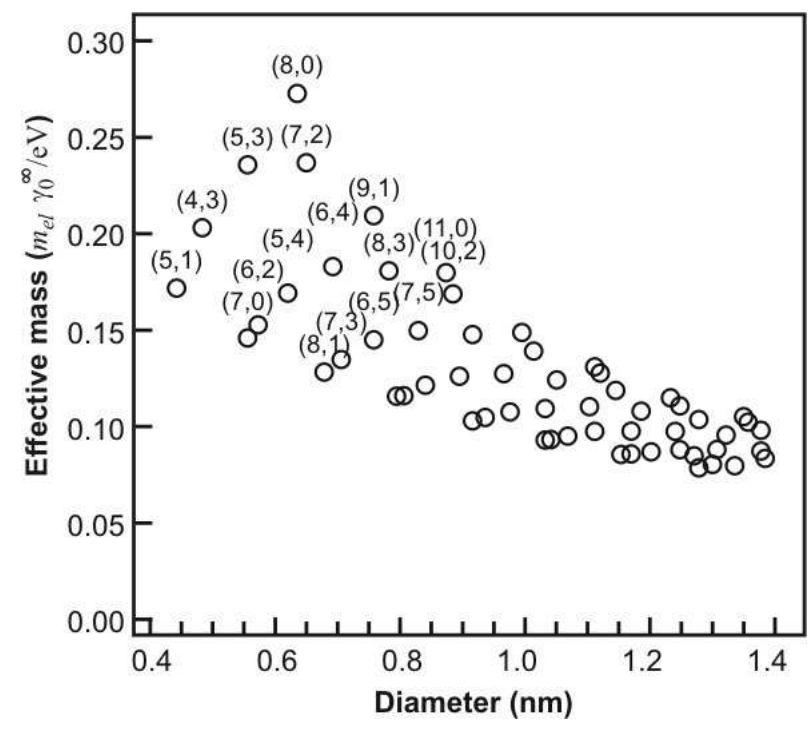

Fig. 4 Effective exciton masses calculated using the chirality and diameter corrected TB-band-structure model [26]. In contrast to expectations for excitonic transitions, there is no apparent correlation of transition energies with effective masses.

where $\epsilon_{11}^{\mathrm{TB}}$ are the TB band-gaps as calculated using the diameter and chirality corrected nearest neighbor hopping integrals [26]. Best agreement with experiment is here obtained for $\gamma_{0}^{\infty}=3.022 \mathrm{eV}, \Omega=0.130 \mathrm{eV}$, $A=-0.0543 \mathrm{eV}$, and $B=0.00395 \mathrm{eV}$. The quality of the agreement between experiment and calculated transition energies is evident from Table 1and Fig. 3id) where calculated gap-energies are plotted as a function of the tube diameter. Note, that the root mean square deviation is below $4 \mathrm{meV}$.

Alternatively, we have also combined diameter and chirality corrected TB calculations with a simple Wannier type exciton model. Transition energies are here calculated within the effective mass approximation allowing also for fractal dimensionality of excitons $\alpha$, with $1<\alpha<3$. The transition energy is then given by:

$$
E_{11}^{S}(n, m)=\gamma_{0}^{\infty} \cdot \epsilon_{11}^{\mathrm{TB}}(n, m)-\frac{\mu E_{H}}{\epsilon^{2} m_{e l}\left(n+\frac{\alpha-3}{2}\right)^{2}}
$$

where $n=1,2,3, \ldots$ is the principal exciton quantum number while $\mu$ and $m_{e l}$ are the effective exciton and free electron mass and $\epsilon$ is the dielectric constant [18]. $E_{H}$ is the Rydberg constant. $\mu(n, m)$ is obtained from TB-valence and conduction band effective masses $m_{h}$ and $m_{e}$ using $\mu^{-1}=m_{e}^{-1}+m_{h}^{-1}$. For semiconducting tubes studied here $\mu$ is found to vary strongly with chirality and diameter and decreases from values around $0.25 m_{e l} \gamma_{0}^{\infty} / \mathrm{eV}$ at $0.6 \mathrm{~nm}$ diameter to about $0.10 m_{e l} \gamma_{0}^{\infty} / \mathrm{e}$ at $1.3 \mathrm{~nm}$ diameter (see Fig. (4). The dielectric constant is assumed to be less dependent on chirality and diameter and the exciton binding energy should thus be most strongly influenced by its effective mass. Interest- ingly, a fit of experimental data to eq. 2 where $\epsilon$ and $\alpha$ are treated as adjustable parameters provides no significant improvement over the zero-order one-parameter TB model described above. We also find no correlation of any of the residuals described above with $\mu(n, m)$.

This analysis suggests that optical transitions in SWNT micelles are not excitons of the Wannier type. However, other observations are in favor of such an interpretation as for example the large value of $\gamma_{0}^{\infty}=3.438 \mathrm{eV}$ in the one-parameter fit. It can hardly be reconciled with previously reported values. In particular band-gaps observed in scanning tunneling spectroscopy are at variance with the transition energies measured here. The band-gap measured by STS is determined by the threshold for injection of free electrons or holes into the bandstructure of SWNTs and Coulomb interactions play a comparatively negligible role. However, Coulomb interactions are crucial for exciton formation and lead to a renormalization of the band-structure obtained from non-interacting electron models such as the TB calculation. The general trend of Coulomb interactions would be to significantly raise the energy needed for excitation of free electron-hole $(e-h)$ pairs. The discrepancy between transition energies observed in optical and STS experiments would thus be easily resolved if optical transitions in SWNTs were excitonic in character. A comparison of the high transition energies observed here for a specific tube type with results from STS experiments, suggests that Coulomb interactions lead to a substantial renormalization of the non-interacting electron bandstructure and gap energies perhaps by as much as ten to tens of percent. Similar changes of band-gap energies as a consequence of Coulomb interactions have been predicted theoretically by Ando 33 .

Here, a further point of interest is the observation that the absorption spectrum of Fig. B $\mathrm{k}$ ) can be extremely well described by a linear combination of nearly perfectly symmetrical absorption features. Note, that absorption by generation of free electron-hole pairs would be associated with a pronounced asymmetry of absorption features. Specifically, the joint density of states (JDOS) shows such asymmetries and consequently also the absorption cross sections - even if spectral broadening is included 13 22]. The highly symmetrical shape of absorption features thus appears to be consistent with excitonic transitions.

In conclusion, we observe striking agreement of experimental transition energies and energies obtained from a semi-empirical 4 parameter model based on refined TB band-structure calculations. This strongly suggests that the observed oscillation of transition energies with diameter and chirality is dictated by corresponding oscil$\mathrm{V}$ lations of the TB band-gaps. The somewhat unexpectedly large band-gaps and the highly symmetrical shape of absorption features in NIR spectra are consistent with an excitonic character of optical transitions. We speculate, that the offset parameter $\Omega$ provides a rough mea- 

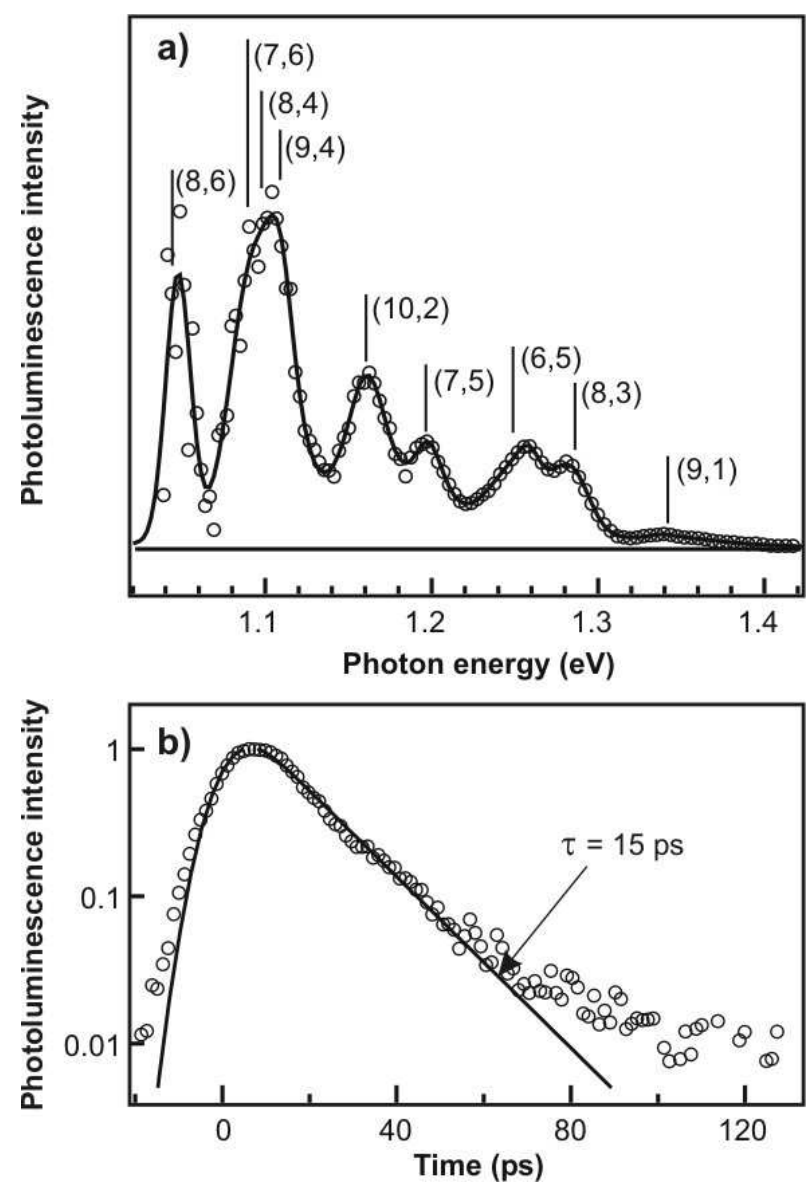

Fig. 5 a) Photoluminescence spectrum from individual SWNT-micelles. The name and diameter of a number of tubes is also specified. b) Energy integrated photoluminescence decay. The decay has been fit by a 15 ps decay (solid line).

sure of the difference between band-gap widening due to Coulomb interactions and the exciton binding energy. This would also suggest that exciton binding energies are only weakly dependent on tube diameter and chirality, i.e. their effective mass, which appears to be at variance with expectations for Wannier type excitons. The nature of optical transitions in the band-gap region thus remains somewhat unclear and further experimental work seems necessary to unambiguously identify the character of optical excitations in SWNTs.

\subsection{Charge-carrier dynamics in individual SWNTs}

In the following we will discuss scattering and relaxation processes of optically excited carriers in individual SWNTs and SWNT ropes as studied by time-resolved photoluminescence (PL) and photoelectron (PE) spectroscopy.

If SWNTs are exfoliated from ropes and dispersed in aqueous solution one finds that the PL quantum yield increases by orders of magnitude reaching values on the or-

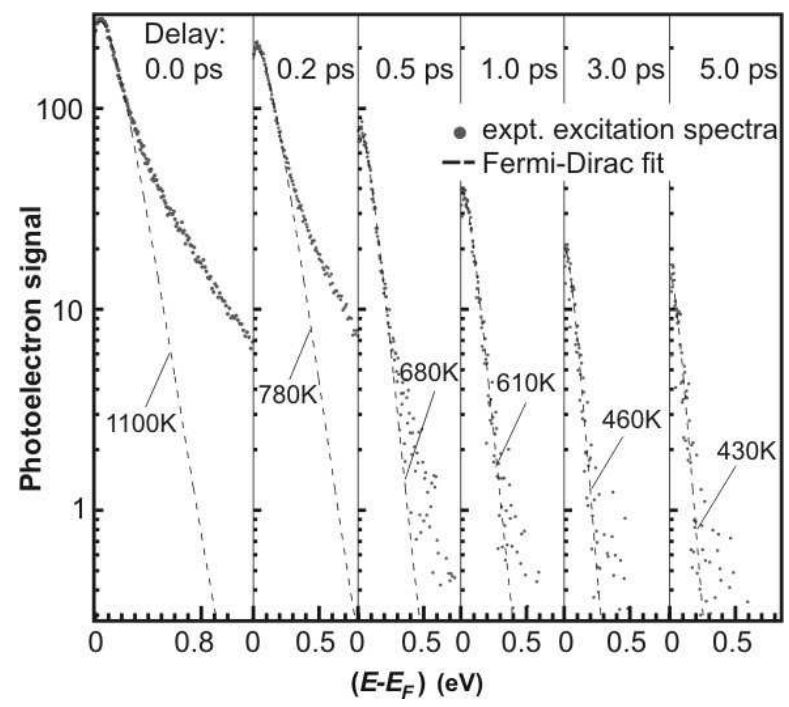

Fig. 6 Femtosecond time-resolved photoelectron spectra at different time-delays up to $5 \mathrm{ps}$. The contribution of photoexcited non-thermal electrons is evident from the apparent deviation of the experimental data from the Fermi-Dirac fit (dashed line), in particular at early delay times up to about $0.5 \mathrm{ps}$

der of $10^{-3}$. 15] The associated PL spectra are characterized by a very small Stokes shift of about $5 \mathrm{meV}$. In Fig. [5 a) we have plotted a PL spectrum from water suspended $\mathrm{HiPCO}$ micelles in the wavelength range from $850 \mathrm{~nm}$ to $1200 \mathrm{~nm}$. The pump power in these experiments corresponds to a pulse fluence of below $\cdot 10^{13}$ photons per $\mathrm{cm}^{2}$ such that nonlinear effects are not expected. The major features can be linked to absorption features in the same energy range corresponding to transitions in tubes with small diameters between 9.1 Åand $7.6 \AA$. We have marked the peak positions and diameters of a few tubes in this energy range. The energy-integrated PL signal in Fig. (5) decays with a time-constant of about 15 ps. Deviations from a simple exponential decay may be indicative of a distribution of relaxation times in these samples, i.e. heterogeneous broadening of the corresponding transitions. Such heterogeneities may arise, for example, from a distribution of tube lengths. In combination with the recently reported PL quantum yield of $10^{-3}$ [15. This indicates that the radiative lifetime of the luminescent states is on the order of $10 \mathrm{~ns}$. The fast decay observed here thus underlines the importance of nonradiative decay processes for the PL lifetime. The detailed mechanism of such non-radiative decay processes, however, needs to be explored in further detail.

\subsection{Charge-carrier dynamics in $S W N T$ ropes}

As mentioned above, the luminescence quantum yield is reduced severely if tubes are agglomerated in tube ropes were tube-tube interactions lead to a rapid decay of excited states. This is also supported by the $\approx 100 \mathrm{meV}$ 


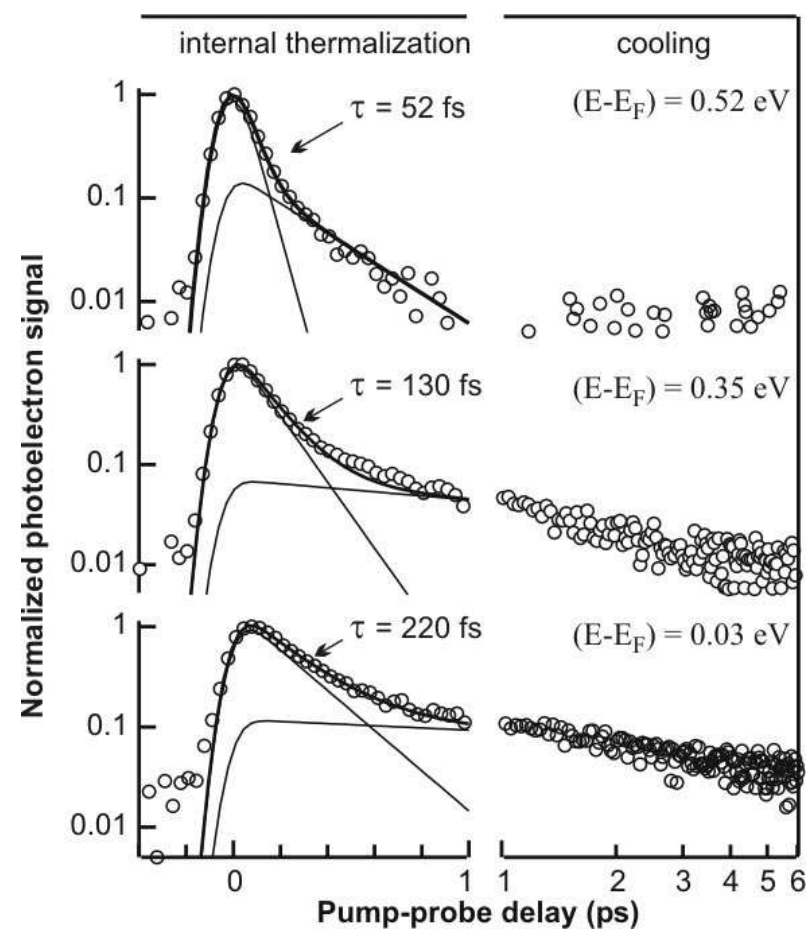

Fig. 7 Photoelectron cross-correlation traces from SWNT ropes for three different electron energies. The initial fast component is seen to be strongly energy dependent and can be associated with electron-electron scattering leading to rapid internal thermalization of the laser excited system. The slow component is associated with electron-phonon interactions and leads to a somewhat slower cooling of the system back to the lattice temperature of $300 \mathrm{~K}$.

increase of linewidths in absorption spectra from crystalline SWNT samples [13. The fast decay of excited states can be clearly seen in femtosecond time-resolved photoelectron spectra as the ones shown in Fig. [6 the photoelectron signal from the vicinity of the Fermi level of SWNT ropes is shown on a logarithmic scale for different pump-probe delays. Here, the system was perturbed by a visible $2.32 \mathrm{eV}$ pump pulse and was probed by a weaker second harmonic $4.64 \mathrm{eV}$ UV probe pulse. The photoelectron spectra clearly show the dynamics of the optically excited SWNT ropes on the sub- and picosecond timescales. The absence of pronounced features in these spectra similar to those found in optical spectra can be attributed to the greater sensitivity of photoemission to the alignment of the band-structure with respect to the Fermi level [4,7.

If spectra are fit by a hot Fermi-Dirac distribution (dashed lines) one finds that the electron distribution has a non-thermal component up to a pump-probe delay of about $0.5 \mathrm{ps}$. Here, the process of internal thermalization of the electronic system after photoexcitation can be characterized by a time-constant of $200 \mathrm{fs}$. We have previously shown that the latter can be attributed to rapid electron-electron scattering processes similar to those in graphite [4]7. At the same time one also finds that after

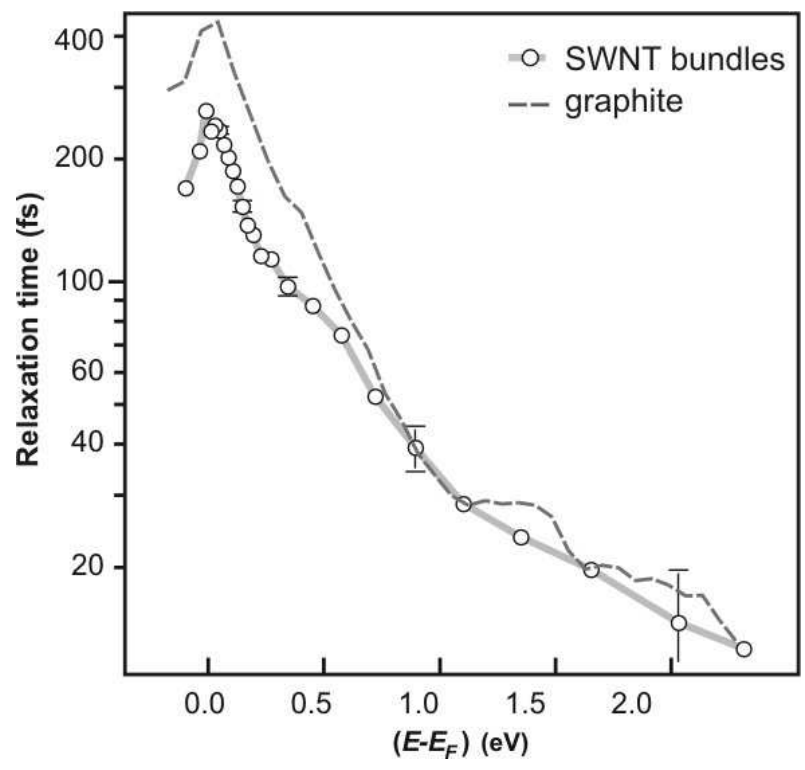

Fig. 8 Energy dependence of the fast component in crosscorrelation traces from SWNT ropes. The pronounced increase of relaxation times near the Fermi-level and the resemblance with results in graphite suggest that the fast component is associated with electron-electron scattering processes.

a rapid initial jump to slightly above $2000 \mathrm{~K}$ the temperature of the electronic system cools back down towards the lattice temperature of $300 \mathrm{~K}$ on the picosecond time-scale. This process is intimately related to the electron-lattice interaction and can be used to quantitatively determine the electron-phonon mass enhancement parameter [3].

The dynamics of internal thermalization and cooling can also clearly be discriminated using the timedependence of the intensity at a specific photoelectron energy (see Fig. 17). Each of the cross-correlation traces in Fig. 7 monitors the intensity within a certain energy window in the PE-spectrum. Using the probe photon energy the latter can be related to a specific intermediate state energy with respect to the Fermi level. The cross-correlation traces in Fig. [7 can be decomposed into two components, one decaying on the subpicosecond and one on the picosecond time-scale. The fast component is associated with the internal thermalization of the electronic system while the slow component is related to the cooling of the laser heated system [7].

The energy dependence of the fast component is plotted in Fig. 8 showing a pronounced increase of relaxationtimes in the vicinity of the Fermi-level, a behavior typical of metallic systems 34. Similar decay times are also found in graphite [35] (see Fig. 8) where the fast decay could be attributed to intralayer electron-electron scattering by a comparison with recent ab inito selfenergy calculations 35 36. The fast sub-picosecond decay found here can readily account for the vanishingly small PL quantum yield observed for SWNT ropes. The decay of excited states in semiconducting tubes is be- 


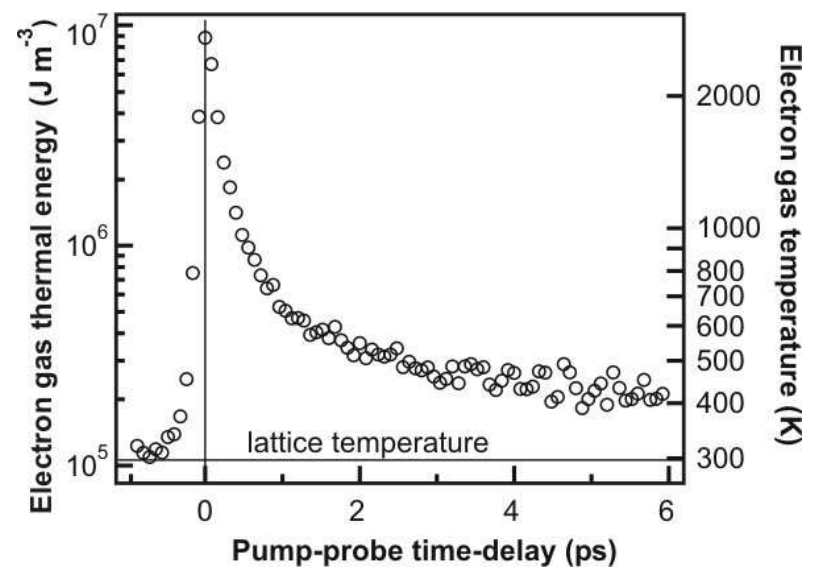

Fig. 9 Evolution of the internal energy and the corresponding electron gas temperature of the electronic system after laser heating.

lieved to be facilitated by rapid intertube electron scattering and successive generation of low energy secondary electron cascades, primarily in metallic tubes. The excess energy of the resulting hot electron distribution is finally distributed among lattice and electronic degrees of freedom by the $e-p h$ interactions discussed in the following parahraphs.

The slow decay seen in the cross-correlation traces of Fig. 7 is analyzed in more detail following the approach described in ref. [14. To illustrate this in detail, we have plotted the electronic temperature $T_{e}$ and the corresponding internal energy of the electronic system as a function of time before and after laser excitation in Fig. 9 The internal energy of the electronic system is seen to jump to about $10^{7} \mathrm{~W} / \mathrm{m}^{3}$ as a consequence of laser excitation. If the evolution of the internal energy is differentiated with respect to time one obtains the rate of energy change of the electronic system $H=H\left(T_{e}, T_{l}\right)$, or more specifically the rate at which energy is transferred between electrons at temperature $T_{e}$ and the lattice at temperature $T_{l}$ (see inset of Fig. 9). This rate can be related to the electron-phonon mass-enhancement parameter $\lambda$ using:

$$
H\left(T_{e}, T_{l}\right)=\frac{144 \zeta(5) k_{B} \gamma}{\pi \hbar} \frac{\lambda}{\Theta_{D}^{2}}\left(T_{e}^{5}-T_{l}^{5}\right)
$$

where $\zeta(5)=1.0369 \ldots$ is Riemann's Zeta function, $\gamma$ is the specific electronic heat capacity and $\Theta_{D}$ the samples Debye temperature 14,37. Note, that we here measure the electron-phonon energy transfer in metallic tubes because metallic tubes contribute most strongly to the density of states near the Fermi level. For the following analysis we used a Debye temperature of $1000 \mathrm{~K}$ and a specific heat capacity of nanotube ropes with a 2:1 mixture of semiconducting to metallic tubes of $12 \mu \mathrm{J} / \mathrm{mole} / \mathrm{K}$. A fit to the temperature dependence of the experimental energy transfer rate using the above constants yields an extraordinary small $\lambda$ of $0.0004 \pm 0.0001$ (see Fig. 9). This

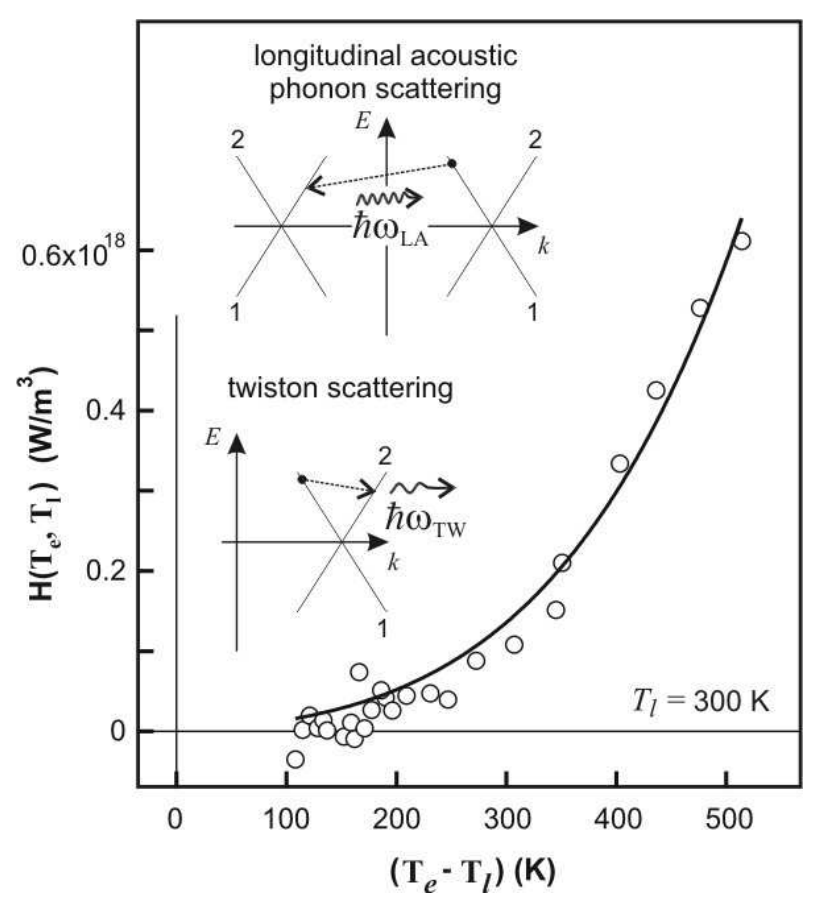

Fig. 10 Dependence of the electron-to-lattice energy transfer rate of metallic tubes in tube ropes on the electron gas temperature. The lattice temperature in this experiment was $300 \mathrm{~K}$. The solid line is a fit to eq. 3] used to determine the strength of the electron-phonon coupling constant $\lambda$. Also included is a schematic representation of two conceivable scattering processes with twistons or longitudinal acoustic phonons.

weak coupling is consistent with electron scattering from to two different acoustic phonon modes: a longitudinaland a so called twiston mode 3 . Note that the electronphonon mass enhancement in copper is almost 3 orders of magnitude higher 38 .

The above value for $\lambda$ can also be used to calculate the ballistic $e-p h$ mean free path from the $e-p h$ scattering time $\tau_{e-p h}$ which is given by:

$$
\frac{1}{\tau_{e-p h}}=24 \pi \zeta(3) \frac{k_{B}}{\hbar} \frac{\lambda}{1+\lambda} \frac{T_{e}^{3}}{\Theta_{D}^{2}}
$$

With $\zeta=1.2020 \ldots$ and the above mass enhancement parameter this would give a room temperature scattering time in metallic nanotubes of $15 \mathrm{ps}$ which corresponds to an $e-p h$ mean free path $l_{e-p h}$ of $15 \mu \mathrm{m}$ [39. This provides a clear confirmation of previously reported long $e$ - $p h$ ballistic mean free paths [40 41] from the perspective of a time-domain experiment.

\section{Conclusions}

We have studied the electronic structure and dynamics of optically excited SWNTs in crystalline ropes as well as isolated in solution. The transitions in optical spectra 
of individual SWNTs can be well described by a semiempirical, tight-binding based model with four free parameters. Our analysis of optical spectra shows that the variation of transition energies with tube diameter and chirality can be attributed to oscillations of the band-gap obtained from a refined tight-binding calculation. Note, that fully empirical expressions of transition energies of the type given in ref. 16 are very useful for the interand extrapolation of band-gaps to presently unobserved tube species but provide little insight into the physical origin underlying band-gap variations if different tube types are compared. One of the corrective terms in the semi-empirical expression presented here, for example, clearly identifies a chirality dependent contribution to transition energies whose origin yet needs to be clarified. Furthermore, in spite of the evidence in favor of an excitonic character of optically excited states we find no correlation of the observed transition energies with effective electron or hole masses as expected for WannierMott type excitons.

The dynamics and energy relaxation in optically excited tube ropes is found to be dominated by rapid intraand intertube scattering processes that lead to internal thermalization of the laser-heated electronic system within about $200 \mathrm{fs}$, most likely through electron-electron interactions. The coupling of the hot electron gas to phonons via scattering from longitudinal acoustic phonons or twistons is found to be characterized by a room temperature electron-phonon scattering time on the order of 15 ps. The latter is a consequence of the weak electronlattice coupling also reflected by the experimentally determined $e$-ph mass enhancement parameter $\lambda$ of 0.0004 . Relaxation of photo-excited states near the band-gap of exfoliated semiconducting tubes is significantly slower than in ropes as reflected by the commonly observed increase of photoluminescence quantum yields in isolated tubes to values on the order of $10^{-3}$. Here, we found the luminescence decay of SWNTs in sodium cholate micelles to be about 15 ps which clearly illustrates that nonradiative decay processes dominate energy relaxation in semiconducting SWNTs. This also allows to estimate the radiative lifetime in semiconducting SWNTs to be on the order of $10 \mathrm{~ns}$.

\section{Acknowledgements}

It is our pleasure to acknowledge continuing support by G. Ertl. We also would like to thank G. Dukovic and L. Brus for sharing experimental absorption spectra prior to publication. We also thank M. Antonietti as well as O. Bogen and H. Otto for providing infrastructure for processing nanotube dispersions and I. Dorbandt for making spectroscopic facilities available to us.

\section{References}

1. J. A. Misewich, R. Martel, Ph. Avouris, J. C. Tsang, S. Heinze, and J. Tersoff, Science 300, (2003) 783.

2. M. Freitag, Y. Martin, J. A. Misewich, R. Martel, Ph. Avouris, Nano Letters 3, (2003) 1067.

3. T. Hertel and G. Moos, Phys. Rev. Lett. 84, (2000) 5002-5005.

4. T. Hertel and G. Moos, Chem. Phys. Lett. 320, (2000) 359-364.

5. E. J. Mele, P. Kral, and D. Tomanek, Phys. Rev. B 61, (2000) 7669.

6. X. Sun, Y. N. Xiong, P. Chen, J. Y. Lin, W. Ji, J. H. Lim, S. S. Yang, D. J. Hagan and E. W. Van Stryland, Appl. Optics 39, (2000) 1998-2001.

7. T. Hertel, R. Fasel and G. Moos, Appl. Phys. A-Mater. Sci. Process. 75, (2002) 449-465.

8. L. Vivien, P. Lancon, D. Riehl, F. Hache and E. Anglaret, Carbon 40, (2002) 1789-1797.

9. T. I. Jeon, K. J. Kim, C. Kang, S. J. Oh, J. H. Son, K. H. An, D. J. Bae and Y. H. Lee, Synth. Met. 135, (2003) 429-430.

10. C. L. Kane, E. J. Mele, Phys. Rev. Lett. 90, (2003) 207401.

11. J. S. Lauret, C. Voisin, G. Cassabois, C. Delalande, P. Roussignol, O. Jost and L. Capes, Phys. Rev. Lett. 90, (2003) .

12. S. Lebedkin, F. Hennrich, T. Skipa, and M. M. Kappes, J. Phys. Chem. B 107, (2003) 1949.

13. A. Hagen and T. Hertel, Nano Lett. 3, 2003383.

14. G. Moos, R. Fasel and T. Hertel, J. Nanosci. Nanotechnol. 3, (2003) 145-149.

15. M. J. OConnell, S. M. Bachilo, C. B. Huffman, V. C. Moore, M. S. Strano, E. H. Haroz, K. L. Rialon, P. J. Boul, W. H. Noon, C. Kittrell, J. Ma, R. H. Hauge, R. B. Weisman, R. E. Smalley, Science, 279, 593, (2002).

16. S. M. Bachilo, M. S. Strano, C. Kittrell, R. H. Hauge, R. E. Smalley and R. B. Weisman, Science 298, (2002) 2361-2366.

17. R. Loudon, Am. J. Phys. 27, (1959) 649.

18. X.-F. He, Phys. Rev. B 43, (1991) 2063.

19. P. Nikolaev, M. J. Bronikowski, R. K. Bradley, F. Rohmund, D. T. Colbert, K.A. Smith, R. E. Smalley, Chem. Phys. Lett. 313, (1999) 91.

20. J. Daniels, C. von Festenberg, H. Raether, and K. Zeppenfeld, Optical Constants of Solids by Electron Spectroscopy, in Springer Tracts in Modern Physics Vol. 54 Springer, Berlin, 1970, pp. 126130.

21. R. Ahuja, S. Auluck, J. M. Wills, M. Alouani, B. Johansson, O. Eriksson, Phys. Rev. B. 55, (1997) 4999.

22. Lin, M. F. Phys. Rev. B 61, (2000) 13153.

23. A. Hartschuh, H. N. Pedrosa, L. Novotny, T. D. Krauss, Science 301, (2003) 1354.

24. E. Gregan, S. M. Keogh, T. G. Hedderman, B. McCarthy, G. Farrell,G. Chambers, H. Byrne, J. AIP Conference Proceedings 633, (2002) 294.

25. A. Kukovecz, Ch. Kramberger, V. Georgakilas, M. Prato, H. Kuzmany, Eur. Phys. J. B 28, (2002) 223.

26. J. W. Ding, X. H. Yan, J. X. Cao, Phys. Rev. B 66, (2002) 073401.

27. R. Saito, M. Fujita, G. Dresselhaus, and M. S. Dresselhaus, Phys. Rev. B 46, (1992) 1804. 
28. N. Hamada, S. Sawada, and A. Oshiyama, Phys. Rev. Lett. 68, (1992) 1579.

29. T.W. Odom, J.-L. Huang, P. Kim, and C. M. Lieber, Nature 391, (1998) 62.

30. J. W. G. Wildoer, L. C. Venema, A. G. Rinzler, R. E. Smalley, C. Dekker, Nature 391, (1998) 59.

31. X. Liu, T. Pichler, M. Knupfer, M. S. Golden, J. Fink, H. Kataura,Y. Achiba, Phys. Rev. B 66, (2002) 045411.

32. G. Dresselhaus, M. A. Pimenta, R. Saito, J. C. Charlier, S. D. M. Brown,et al. In: Science and Application of Nanotubes; D. Tomanek, R. J. Enbody, Eds.; Kluwer Academic, New York, 1999; pp 275-295.

33. T. Ando, J. Phys. Soc. Jap. 66, (1997) 1066.

34. T. Hertel, E. Knoesel, M. Wolf, and G. Ertl, Phys. Rev. Lett. 76, (1996) 535.

35. G. Moos, C. Gahl, R. Fasel, M. Wolf and T. Hertel, Phys. Rev. Lett. 87, (2001) 267402.

36. C. D. Spataru,M. A. Cazalilla, A. Rubio, L. X. Benedict, P. M. Echenique,and S. G. Louie, Phys. Rev. Lett. 87, (2001) 246405.

37. P.B. Allen, Phys. Rev. Lett. 59, (1987) 1460.

38. S.D. Brorson, A. Kazeroonian, J. S. Moodera, D.W. Face, T.K. Cheng, E.P. Ippen, M.S. Dresselhaus, and G. Dresselhaus, Phys. Rev. Lett. 64, (1990) 2172.

39. P.B. Allen, Phys. Rev. B 11, (1975) 2693.

40. S. Frank, P. Poncharal, Z. L. Wang, W. A. de Heer, Science 280, (1998) 1744.

41. J. Appenzeller, R. Martel, P. Avouris, H. Stahl, and B. Lengeler, Appl. Phys. Lett. 78, (2001) 3313. 